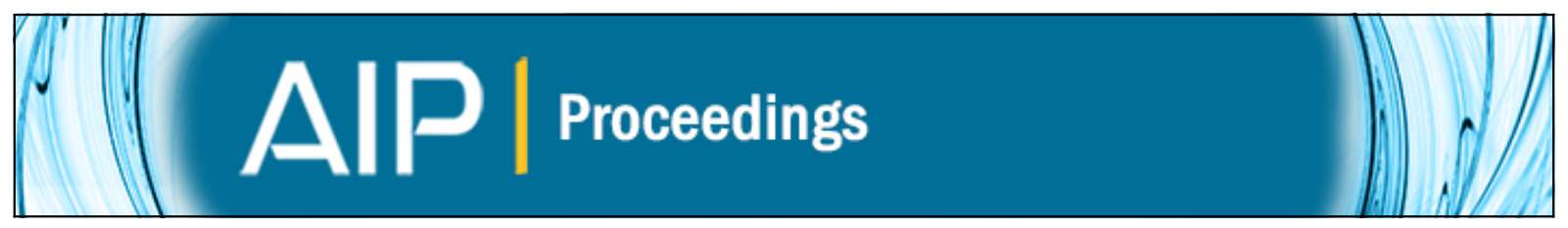

Contribution to the Wednesday afternoon discussion on spectroscopic factors

C. Barbieri

Citation: AIP Conference Proceedings 791, 57 (2005); doi: 10.1063/1.2114693

View online: http://dx.doi.org/10.1063/1.2114693

View Table of Contents: http://scitation.aip.org/content/aip/proceeding/aipcp/791?ver=pdfcov

Published by the AIP Publishing 


\title{
Contribution to the Wednesday afternoon discussion on spectroscopic factors
}

\author{
C. Barbieri \\ TRIUMF, 4004 Wesbrook Mall, Vancouver, British Columbia, Canada V6T 2A3
}

\begin{abstract}
This part of the discussion would like to review the concept of spectroscopic factors and how they relate to measured cross sections and nuclear correlations. A profound knowledge of how correlations affect the spectral function can help to better understand transfer reactions. Nowadays, we have a fairly complete picture for protons in stable nuclei but a lot remain to be learned regarding exotic species.
\end{abstract}

Keywords: Spectroscopic factors, spectral function, nucleon transfer, nuclear correlations PACS:

\section{ON THE MEANING AND USEFULNESS OF SPECTROSCOPIC FACTORS}

Several contributions to the present meeting emphasized that experimental cross sections (for transfer of a nucleon) are on average lower than what is predicted by the independent particle model (IPM). Whence, the need for introducing spectroscopic factors that account for (and provide a measure of) the occupation of the relevant orbitals. However, the attempts to extract experimentally such information have produced discordant results—and a quite a bit of confusionover the years. Still, as not all of the relevant astrophysical reactions can be determined from measurement, it is imperative to understand the mechanisms of such quenching and how to provide reliable predictions of it.

From the theoretical point of view, there exist a precise definition of spectroscopic factors as the norm of the onebody overlap functions,

$$
Z \equiv \int d^{3} r\left|\psi_{n}(r)\right|^{2}, \quad \quad \text { where } \quad \psi_{n}(r)=\left\langle\Psi_{n}^{A-1}\left|a_{\mathbf{r}}\right| \Psi^{A}\right\rangle
$$

and $a_{\mathbf{r}}$ is the operator destroying a particle at position $\mathbf{r}$. If $\left|\Psi_{n}^{A-1}\right\rangle$ and $\left|\Psi^{A}\right\rangle$ were Slater determinants, $Z$ would be equal to 1 and $\psi_{n}(r)$ would be the mean field orbital occupied only in $\left|\Psi^{A}\right\rangle$. However, in Eqs. (1) they represent exact eigenstates of the nuclear Hamiltonian in the complete (A-1)- and A-body Hilbert spaces. In this situation $\psi_{n}(r)$ is the solution of the microscopic optical potential derived from Feshbach theory and the corresponding orbital is depleted to $Z<Z_{I M P}$.

We note that once the nuclear Hamiltonian has been chosen, Eqs. (1) uniquely define the spectroscopic factors. However, one must remember that different models of the NN interaction have different off-shell behaviors while they reproduce the same scattering data. This poses an issue of consistency between the treatment of the initial state (hence the spectroscopic factor), the final state interactions and the interactions with the probe: all these contributions come together to generate the observed cross section and should, in principle, be derived from the same microscopic Hamiltonian [1] - also using consistent electromagnetic currents, in case of electron scattering. This is a formidable problem in many-body nuclear physics, hence one is often forced to model scattering and bound states in terms of Wood-Saxon potentials. In general, a fit to a few Woods-Saxon parameters does not guarantee consistency and the uncertainty increases when the reaction mechanism is only sensitive to the nuclear surface (see, for example, contribution by P. Capel). There are two types of uncertainties here: one due to our incapability of solving exactly the many-body problem and one intrinsic to the unknown off-shell behavior of the nuclear force. Still, for a given realistic $\mathrm{NN}$ interaction, a proper microscopic theory should be able to describe different transfer reactions on a nucleus using the same occupation numbers. That this is possible was shown, for example, by Kramers et al. [2], where $\left(e, e^{\prime} p\right)$ and $\left(d,{ }^{3} \mathrm{He}\right)$ data are reconciled by analyzing both reactions with the same overlap functions. Recently, Ref. [3] achieved a coherent description of initial and final states for ${ }^{16} \mathrm{O}\left(e, e^{\prime} p\right)$, showing that data at very different kinematics can be explained with the same spectroscopic factors-and in particular independently of the $Q^{2}$ transferred by the electron.

We note that the spectroscopic factors extracted from $\left(e, e^{\prime} p\right)$ experiments with different parameterizations of initial and final states tend to agree with each other to within a $10 \%$ of the IPM value. This is possible due to the sensitivity 


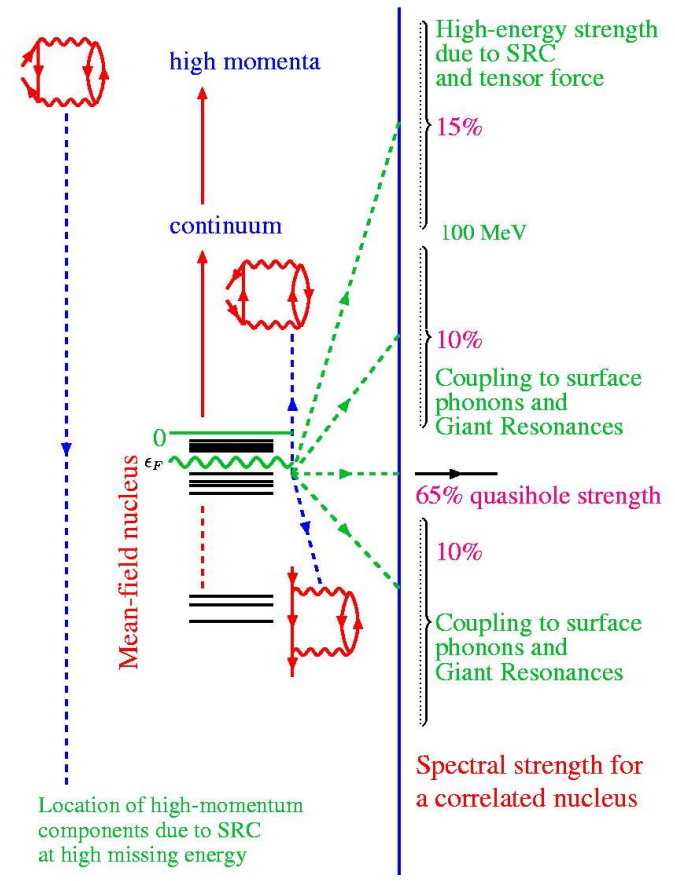

FIGURE 1. Distribution of single particle strength for a nucleus like ${ }^{208} \mathrm{~Pb}$. This applies to an orbital near to the Fermi level.

of the cross section to the overlap function in the interior of the nucleus and to the lower uncertainty on the optical potential for protons emitted at energies of $90 \mathrm{MeV}$ (or larger). A particularly well studied reaction is ${ }^{16} \mathrm{O}\left(e, e^{\prime} p\right)$, for which data exists in different kinematical regimes $[4,5]$ and several theoretical studies have been done, including relativistic effects. The fact that these studies give similar spectroscopic factors is an indication that the uncertainty intrinsic to the nuclear Hamiltonian is contained and that the quenching observed for the quasiparticle peaks hint at actual physics happening in the nucleus.

\section{Nuclear correlations and spectral function}

If one is to make predictions for the quenching of the occupation of a given orbital, he/she will be facing the questions of where the missing strength goes and what are the possible mechanisms (or correlations) that induce its distribution. A general picture of the correlations in nuclei can be achieved by considering the one-body spectral function [6]

$S(r, \omega)=S^{h}(r, \omega)+S^{p}(r, \omega)=\sum_{n}\left|\left\langle\Psi_{n}^{A-1}\left|a_{\mathbf{r}}\right| \Psi^{A}\right\rangle\right|^{2} \delta\left(\omega-E^{A}+E_{n}^{A-1}\right)+\sum_{n}\left|\left\langle\Psi_{n}^{A+1}\left|a_{\mathbf{r}}^{\dagger}\right| \Psi^{A}\right\rangle\right|^{2} \delta\left(\omega-E_{n}^{A+1}+E^{A}\right)$

which, for transitions to discrete states, contains the same information of Eqs. (1). The hole part of the spectral function, $S^{h}(r, \omega)$, is interpreted as the probability of finding a nucleon with energy $\omega$ at position $\mathbf{r}$. Analogously, $S^{p}(r, \omega)$ is the probability of adding a nucleon. In spite these two quantities refer to different processes (knock out and capture), it must be remembered that they are intimately related through the Pauli principle, namely, by the sum rule

$$
\int_{-\infty}^{\infty} d \omega S(r, \omega)=1
$$

This imply that small probabilities of extracting a nucleon (low occupation) leave room to large cross sections for capturing one to the same orbital.

The characteristics of the nuclear spectral function of protons have been extensively investigated in recent years for nuclei along the stability line [7]. Electron scattering experiments have shown that in closed shell mean field orbits are 
TABLE 1. Dependence of theoretical spectroscopic factors (as a fraction of the IPM value) on the inclusion of various short- and long-range correlations (LRC) effects.

\begin{tabular}{lcc}
\hline Spect. factors of ${ }^{16} \mathbf{O}$ & $Z_{p 1 / 2}$ & $Z_{p 3 / 2}$ \\
\hline SRC only [10, 13] & $\sim 0.90$ & $\sim 0.90$ \\
SRC + LRC(RPA) [14] & 0.74 & 0.72 \\
SRC + LRC(RPA + sp dressing) [14] & 0.77 & 0.72 \\
experiment [3, 15] & $0.64-0.71$ & $0.54-0.61$ \\
\hline
\end{tabular}

indeed well defined near to the Fermi level, however, these have spectroscopic factors of the order of $60 \%$ of the IPM value [8]. Orbitals at larger energies are spread over a wider range of energies due to the coupling to $2 \mathrm{~h} 1 \mathrm{p}(2 \mathrm{p} 1 \mathrm{~h})$ states and to the continuum. Here, occupation numbers are no longer a well defined concept. Still, one can approximately think in terms of occupation by integrating the strength function, Eq. (2), over a proper range of energies.

Short-range correlations (SRC) are induced by the repulsive core at small distances and by the tensor operator present in the NN potential. Different theoretical evaluations of SCR suggest that these move about 10-15\% of the total strength to very high momenta and energies $[9,10]$. The experimental observation of such effects has been a challenging issue for many years and could be achieved only recently at Jefferson Lab [11]. In this experiment, the high energy of the electron and the emitted proton allow for for a semiclassical analysis of the reaction mechanism [12], which supports the evidence for the presence of high momentum components in the nuclear wave function.

Figure 1, gives a qualitative sketch of the strength distribution of Eq. (2) for an orbit just below the Fermi energy, together with the self-energy diagrams responsible for spreading it in the particle and hole domains. The IPM is depicted in the central column. The contributions to the reduction of spectroscopic factors are summarized in table 1 for the $p$ shell orbitals of ${ }^{16} \mathrm{O}$. A large part of the quenching can be described as coupling of single particle motion to collective surface phonons [14]. However, the dressing of quasiparticles partially screens these correlations. This particular nucleus has so far defeated a complete theoretical description. Ref. [14] suggested that this may be due to the poor description of the excitation spectrum in random phase approximation (RPA). Further work is being pursued to clarify this point [16].

The above experimental and theoretical works identify the global properties of protons in nuclei along the stability line[7]. However it is not clear how these features can be extrapolated toward the drip lines. Recent nuclear break up experiments at NSCL have begun providing first information for nucleons (including neutrons) in isotopes near the stability lines[17]. These point, for example, to larger occupation for halo states (see contribution by J. Tostevin). A proper understanding of this information will be crucial to push forward our knowledge of exotic nuclei.

\section{ACKNOWLEDGMENTS}

One of us (C.B.) would like to acknowledge helpful discussions with W. H. Dickhoff, L. Lapikás, B. Jennings, M. Radici, D. Rohe, I. Sick and E. Vogt. The work of C.B. is supported by the Natural Sciences and Engineering Research Council of Canada (NSERC).

\section{REFERENCES}

1. S. Boffi, C. Giusti, F. D. Pacati, and M. Radici, Electromagnetic Response of Atomic Nuclei, Oxford Studies in Nuclear Physics (Clarendon Press, Oxford, 1996); S. Boffi, C. Giusti, and F. D. Pacati, Phys. Rep. 226, 1 (1993).

2. G. J. Kramers, H. P. Blok, and L. Lapikás, Nucl. Phys. A679, 267 (2001).

3. M. Radici, W. H. Dickhoff, and E. Roth Stoddard, Phys. Rev. C 66, 014613 (2002).

4. M. Leuschner et al., Phys. Rev. C 49, 955 (1994).

5. K. G. Fissum et al. [Jefferson Lab Hall A Collaboration], Phys. Rev. C 70, 034606 (2004)

6. A. L. Fetter and J. D. Walecka, Quantum Theory of Many-Particle Physics (McGraw-Hill, New York, 1971).

7. W. H. Dickhoff and C. Barbieri, Prog. Part. Nucl. Phys. 52, 377 (2004).

8. L. Lapikás, Nucl. Phys. A553, 297c (1993).

9. O. Benhar, A. Fabrocini, S. Fantoni, and I. Sick, Nucl. Phys. A579 493 (1994).

10. H. Müther, A. Polls, and W. H. Dickhoff, Phys. Rev. C 51, 3040 (1995).

11. D. Rohe, et al., Phys. Rev. Lett. 93, 182501 (2004). 
12. C. Barbieri and L. Lapikás, Phys. Rev. C 70, 054612 (2004).

13. D. Van Neck, M.Waroquier, A. E. L. Dieperink, S C. Pieper, and V. R. Pandharipande, Phys. Rev. C 57, 2308 (1998).

14. C. Barbieri and W. H. Dickhoff, Phys. Rev. C 65, 064313 (2002).

15. M. Radici, A. Meucci and W. H. Dickhoff, Eur. Phys.. J. A 17, 65 (2003).

16. C. Barbieri, in preparation.

17. P. G. Hansen and J. A. Tostevin, Ann. Rev. Nucl. Part. Sci. 53, 219 (2003). 